\title{
Gravitons and Loops
}

\author{
Abhay Ashtekar ${ }^{1,2}$, Carlo Rovelli ${ }^{3,4}$ and Lee Smolin ${ }^{1}$ \\ 1 Department of Physics, Syracuse University, Syracuse NY 13244-1130, USA \\ ${ }^{2}$ Max Planck Institut für Astrophysik, D8046 Garching bei München, Germany \\ ${ }^{3}$ Department of Physics, University of Pittsburgh, Pittsburgh PA 15260, USA. \\ ${ }^{4}$ Dipartimento di Fisica, Universita' di Trento, Trento; INFN Sezione di Padova, Italy.
}

\begin{abstract}
The recently proposed loop representation is used to quantize linearized general relativity. The Fock space of graviton states and its associated algebra of observables are represented in terms of functionals of loops. The "reality conditions" are realized by an inner product that is chiral asymmetric, resulting in a chiral asymmetric ordering for the Hamiltonian, and, in an asymmetric description of the left and right handed gravitons. The formalism depends on an arbitrary "averaging" function that controls certain divergences, but does not appear in the final physical quantities. Inspite of these somewhat unusual features, the loop quntization presented here is completely equivalent to the standard quantization of linearized gravity.
\end{abstract}




\section{Introduction}

The standard, perturbative, approaches to quantum gravity begin with the quantization of linearized general relativity. They end, of course, with the discovery that perturbative general relativity is not renormalizable. Recently, a nonperturbative approach to quantum general relativity was introduced [1, 2, 3, 4, 5. The framework is based on canonical quantization but has several new ingredients: the use of (self-dual) connections -rather than metrics- as the basic dynamical variables [1], the introduction of a loop representation of quantum states [6, 3], an extension of the Dirac program of quantization of constrained systems [ [⿴囗十, and certain techniques to deal with diffeomorphism invariant quantum field theories [2, 3].

In this paper we return to the problem of the quantization of linearized general relativity and show how the new framework, which has proved to be useful at the nonperturbative level, may be applied to this case as well. More specifically, we present a quantization of linearized general relativity based on the use of the new canonical variables [1] and the loop representation [6, 3]. This is a necessary step in an ongoing program whose aim is to resolve the question of whether the formulation can lead to a successful quantum theory of gravity by resolving the short distance problems nonperturbatively. More specifically, there are three reasons that motivate this investigation.

First, the physical interpretation of the mathematical notions that naturally arise in the exact quantum theory is often rather obscure. For example, in the full theory, physical quantum states arise as suitable functions of knot and link classes of closed curves on a 3-manifold. Compared to the space of states that one normally encounters in physical theories, this space is rather unusual. In order to extract the physical content of these states, it would be extremely useful to relate them to familiar notions such as gravitons. The

first step towards this goal is to recast the Fock description of graviton also in terms of closed loops. This step is completed in this paper.

The second motivation is the following. Since all the techniques used in the non-perturbative approach are novel, it is important to test them in situations in which we have good intuition about the physics of the problem. Over the last two years, therefore, these techniques have been applied to a number of examples which mimic various features of full general relativity. This paper is a continuation of that program. Roughly speaking, the 
examples analyzed so far fall into two categories: those that share certain non-linear features with general relativity, particularly the presence of constraints which are quadratic in momenta and the absence of a background space-time metric [7, 8, 4] and those that test the quantization program in the context of field theories in Minkowski space [9, 10, 5]. In this paper, we will be able to test certain other features of the program which are specific to $3+1$ gravity. These include the use of a hybrid pair of canonical variables in which the configuration variable is complex but the momentum is real, and the strategy of basing the quantization procedure on the "loop algebra" constructed from holonomies of self dual connections and spatial triads.

The third reason that motivates this work arises from the fact that two of the postulates of the standard quantization of free field theories involve notions that cannot be extended to nonperturbative quantization of diffeomorphism invariant theories: the use of the positive/negative frequency splitting to define the vacuum state and operator ordering and the use of the Poincaré group to select the inner product. In the quantization presented here, these are replaced by notions that do extend directly to non-perturbative general relativity - the splitting into self dual and anti-self dual fields rather than positive and negative frequency fields [11], and the use of the "reality conditions" rather than Poincaré invariance to select the inner product. The fact that quantization of the linear theory can be carried out successfully with these replacements provides further confidence in the program as a whole.

Our final description of linearized gravity has two curious features. The first is that the framework has a chiral asymmetry: the left handed gravitons are described in a different way from the right handed ones. The origin of this asymmetry lies in the use of the new canonical variables where the configuration piece is (the restriction to a spatial slice of) the self dual or the anti-self dual part of the spacetime spin-connection. Thus the asymmetry is not an artifact of the linearization; it is simply a consequence of replacing the positive/negative frequency splitting with an ordering prescription that is well defined at both the linearized and non-perturbative level. In spite of the use of one or the other handed connection, however, the final theory contains quanta of both helicities and is completely equivalent to the standard quantum theory of spin two, rest mass zero gravitons (essentially because we now allow both the positive and negative frequency connections [11]). There exist in the literature other frameworks aimed at describing the dynamics of 
(the real, Lorentzian) general relativity which are also intrinsically chirally asymmetric: Penrose's twistor program |12 and the Kozameh-Newman formalism based on lightcone cuts [13]. The detailed relation between the three frameworks, however, remains unclear

The second peculiar feature of our description of linearized gravity is that in the final picture one has to introduce an arbitrary function $f_{r}(\vec{x})$ for averaging (or smearing) loop dependent quantities on a small tube around the loop itself. This is necessary in order to control the infinities that otherwise arise in certain loop integrals. (These infinities could not be cured by a standard regularization technique.) The resulting formalism thus contains a "momentum damping". However, it is exact: no limit or renormalization are needed, and the physical quantities do not depend on the specific choice of the function. Since the technology needed in this averaging procedure tends to obscure the logic of the rest of the paper, we will proceed in two steps. First we ignore the averaging and (formally) construct the entire theory without it. Then we note the appearance of the divergences and repeat the derivation with the averaging. This step will simply amount to replacing certain functions with their averages.

Section 2 discusses the classical features the linearized theory; the Hamiltonian formulation in terms of the new canonical variables is recalled and the classical loop algebra is introduced. Quantization is carried out in section 3 ; the loop representation is constructed, the physical states are isolated by solving the quantum constraints, the correct scalar product is singled out using the "reality conditions" and expressions of certain physical observables are written down explicitly. We conclude in section 4 with a brief discussion of several conceptual issues.

There is a certain number of papers closely related to the present one. The Hamiltonian formulation of linearized gravity in terms of the new canonical variables is discussed in detail in [14]. The loop representation for Maxwell electrodynamics is constructed in detail in [9]. This is the simplest example of a loop representation of a field theory and therefore well-suited to discuss a number of subtleties such as the differences between the use of positive frequency and self dual connections in the construction of the loop algebra and in the physical interpretation of the basic operators on loop states. The connection representation of linearized gravity is constructed and its relation to the loop representation is discussed in [15. (These results are reviewed in 
[ [⿴囗十, 5].) The truncation of the theory obtained by taking the limit of Newton's gravitational constant $G$ to zero, a truncation related but inequivalent to the linearization considered here, is described in reference [16].

\section{Classical theory}

This section is divided into four parts. In the first, we recall (from [14]) the phase space description of linearized general relativity in terms of the new canonical variables; in the second we isolate the true degrees of freedom of the theory; in the third, we present the reality conditions which need to be imposed to ensure that we are dealing with the real gravitational field; and, in the fourth we construct the classical loop algebra for the linearized theory. This algebra will serve as the point of departure for the quantum description in the next section.

\subsection{Linearized gravity in new canonical variables}

The new canonical variables for full (real) general relativity consist of a pair, $\left(A_{a i}, E^{a i}\right)$, of fields on a 3 -manifold $\Sigma$, where $A_{a i}$ is a complex-valued $S O(3)$ connection and $E^{a i}$ is a frame field with density weight one. (Here $a, b, \ldots=$ $1,2,3$ are space indices and $i, j, \ldots=1,2,3$ are internal indices. Unlike in the previous work, for notational simplicity, we will not use tildes to denote the density weights since they play a minimal role in this analysis.) To linearize the theory about flat space, we choose a background point $\left(E^{a i}=E_{o}^{a i}, A_{a i}=\right.$ $0)$ in the phase space where $E_{o}^{a i}$ is a flat triad and consider weak fluctuations around it. Let us begin with the triad. We set:

$$
E^{a i}=E_{o}^{a i}+e^{a i}
$$

Since the background triad is flat, so is the metric $q_{o}^{a b}:=E^{a i} E^{b j} \delta_{i j}$ constructed from it. For simplicity, we will use a set of Cartesian coordinates adapted to it so that we have: $E_{o}^{a i}=\delta^{a i}$. Now the background metric $q_{o}^{a b}$ also has components $\delta^{a b}$ and its determinant is just 1. In what follows, we will often use the background triad to freely interchange the internal and space indices and suitable powers of the determinant of the background met-

ric to balance density weights in various equations. In terms of the perturbed 
triad, the usual linear graviton field, $\gamma_{a b}$, that represents the metric fluctuations around the flat background is given by:

$$
-\gamma^{a b}+\delta^{a b} \gamma_{c}^{c}=2 e^{(a b)}=: h^{a b}
$$

where we have defined $h^{a b}$ as the symmetric part of $e^{a b}$. (The minus sign arises because $e^{a i}$ represents the fluctuation in the contravariant rather than covariant triad and, the trace term, because $e^{a i}$ is a density of weight 1.) Let us next consider the fluctuation in the connection. Since its background value is chosen to be zero, we do not need to introduce a new symbol for its fluctuation. The fundamental (non-vanishing) Poisson bracket of the linearized theory is

$$
\left\{A_{b j}(x), e^{a i}(y)\right\}_{L}=\imath \delta_{b}^{a} \delta_{j}^{i} \delta^{3}(x, y),
$$

where, and in what follows, the subscript or the superscript $L$ denotes structures and quantities of the linearized theory. The linearized theory can now be obtained by expanding the triad using (1) and keeping in all field equations -the constraints as well as the equations of motion- terms which are linear in $e^{a i}$ and $A_{a i}$.

Let us begin by recalling the constraints of the full theory. We have three constraints: a Gauss constraint $\mathcal{G}^{i}=\mathcal{D}_{a} E^{a i} \equiv \partial_{a} E^{a i}+G \epsilon^{i j k} A_{a j} E_{k}^{a}=$ 0 , a vector constraint $\mathcal{V}_{a}=-i E^{b i} F_{a b i}=0$, and a scalar constraint $\mathcal{S}=$ $\epsilon_{i j k} E^{a i} E^{b j} F_{a b}^{k}=0$, where the field strength $F_{a b}^{i}$ is defined by: $F_{a b}^{i}=\partial_{a} A_{b}^{i}-$ $\partial_{b} A_{a}^{i}+G \epsilon^{i j k} A_{a j} A_{b k}$. The linearization of the Gauss constraint yields:

$$
\mathcal{G}_{L}^{i}=\left(\partial_{a} e^{a i}+G \epsilon^{i j a} A_{a j}\right)=0 .
$$

Note that, in spite of the second term in $\mathcal{G}_{L}^{i}$ the algebra of these constraints is Abelian:

$$
\left\{\mathcal{G}_{L}^{i}(x), \mathcal{G}_{L}^{j}(y)\right\}_{L}=0
$$

so that the (internal) gauge group of the linearized theory is $U(1) \times U(1) \times$ $U(1)$. Note that, by contrast, due to the fact that $\mathcal{D}_{a}=\partial_{a}+G A_{a} \times \ldots$, in the full theory we have:

$$
\left\{\mathcal{G}^{i}(x), \mathcal{G}^{j}(y)\right\}=G \epsilon^{i j k} \mathcal{G}^{k}(x) \delta^{3}(x, y)
$$

Therefore, the algebra of the linearized theory can be seen as the $G \rightarrow 0$ contraction of the $S U(2)$ gauge algebra of the full theory. To linearize the 
other two constraints, let us first define the linearized field strength as:

$$
f_{a b}^{i}:=\partial_{a} A_{b}^{i}-\partial_{b} A_{a}^{i}
$$

Note that it is invariant under the linearized gauge transformation, generated by the linearized Gauss constraint $\mathcal{G}_{L}^{i}$. The linearized versions of the vector and the scalar constraints can now be written as:

$$
\begin{gathered}
\mathcal{V}_{a}^{L}:=-\imath f_{a b}{ }^{b}=0, \\
\mathcal{S}_{L}:=-\imath \epsilon_{c}^{a b} f_{a b}{ }^{c}=0 .
\end{gathered}
$$

The constraint algebra is very simple: all of the constraints commute with each other. The variations of the fields $e^{a i}$ and $A_{a}^{i}$ under the gauge transformations generated by the constraints are also simple. Under the canonical transformation generated by the linearized Gauss constraint $\mathcal{G}(\lambda)_{L}:=$ $\int d^{3} x \lambda_{i} \mathcal{G}_{L}^{i}$ we have

$$
\delta A_{a}^{i}=\imath \partial_{a} \lambda^{i}, \quad \delta e^{a i}=-\imath G \epsilon^{a i k} \lambda_{k},
$$

whereas under the linearized vector constraint $\mathcal{V}_{L}(\vec{N}) \equiv \int d^{3} x N^{a} \mathcal{V}_{a}^{L}$ we have:

$$
\delta A_{a}^{i}=0, \quad \delta e_{i}^{a}=\partial_{i} N^{a}-\delta_{i}^{a} \partial_{b} N^{b} .
$$

Notice that in the last equation it is the internal index that becomes the differentiating index in the first term, and that the second term expresses the fact that $e^{a i}$ is a density. Finally, for the scalar constraint $\mathcal{S}(N)_{L} \equiv \int N \mathcal{S}_{L}$ we have

$$
\delta A_{a}^{i}=0, \quad \delta e^{a i}=-2 \epsilon^{b a i} \partial_{b} N .
$$

Let us next consider the Hamiltonian. In the full theory, with asymptotically flat boundary conditions, the Hamiltonian is given by:

$$
H=\frac{1}{2} \int_{\Sigma} d^{3} x N \epsilon^{i j k} F_{a b k} E_{i}^{a} E_{j}^{b}+\frac{1}{2} \oint_{\partial \Sigma} d^{2} S_{b} N \epsilon^{i j k} A_{a k} E^{a i} E^{b j} .
$$

where $N$ is a density of weight minus one. The linearized Hamiltonian can be obtained by first choosing the lapse such that the full evolution keeps the background point $\left(A_{a i}=0, E^{a i}=E_{o}^{a i}\right)$ in the phase space fixed and then keeping terms which are only quadratic in the linearized fluctuations [14]. The 
required lapse in the full theory is just $N=1 / \sqrt{\operatorname{det} q_{o}} \equiv 1$. The linearized Hamiltonian is then given by:

$$
H_{L}=\int_{\Sigma} d^{3} x\left(2 \epsilon_{k}^{i b} f_{a b}{ }^{k} e_{i}^{a}+G\left(A_{a}^{a} A_{b}^{b}-A_{a}{ }^{b} A_{b}{ }^{a}\right)\right) .
$$

In this paper we will use the following boundary conditions on the linearized fields. We assume that $e^{a i}$ falls off as $1 / r$, where $r$ is a radial coordinate in the background metric, while $A_{a i}$ falls off as $1 / r^{2}$. (Usually slightly more stringent conditions are imposed on the fall-off of $e^{a i}$. (See, e.g. [17.) However, our analysis is not sufficiently rigorous to require these refinements.) These ensure that the integral in (14) converges. Note however that the expression itself is not invariant under any of the linearized gauge transformations: the changes it undergoes vanish only when the constraints are satisfied. Thus, the Hamiltonian is unambiguously defined only on the constraint surface. This is of course a general feature of the Dirac analysis of systems with first class constraints; the Hamiltonian is unique only up to addition of constraint functionals. We shall now use this freedom in the definition to re-express the Hamiltonian in a more convenient form.

To do so, let us first define the "magnetic field" $B^{c i}$ of the connection $A_{a}^{i}$ :

$$
B^{c i}=\frac{1}{2} \epsilon^{a b c} f_{a b}^{i}
$$

Using this definition, going to the Fourier components, and using the freedom mentioned above, the linearized Hamiltonian can be now re-expressed in a form that will be more directly useful later on. We have:

$$
H_{L}=-\frac{1}{2} \int d^{3} k\left(2 e_{b a}(-k)-\frac{G}{k^{2}} B_{b a}(-k)\right) B^{a b}(k) .
$$

Using the reality conditions of section 2.3, one can recognize that (16) is just the momentum space version of the expression $H_{L}=\frac{1}{2} \int d^{3} x \bar{A}_{a}^{i}(x) A_{i}^{a}(x)$ in terms of the self-dual connection and its complex conjugate given in [1]. The fact that the form of the Hamiltonian is rather curious has nothing to do with gravity; it is a consequence only of having written the theory in terms of the self-dual connection. For example, the Hamiltonian of the Maxwell theory has a completely analogous form, $H=\int d^{3} x \bar{B}_{+}^{a}\left(B_{+}\right)_{a}$, when written in terms of the self-dual "magnetic field" $B_{+}^{a}=B^{a}+\imath E^{a}$ and its complex conjugate [9]. 
To complete the classical description, it only remains to specify the reality conditions. As mentioned above, these will be discussed in section 2.3. In the next sub-section, we make a brief detour to recall how to extract the physical degrees of freedom in the Hamiltonian formulation of the classical theory.

\subsection{Physical degrees of freedom}

We review from [14] the analysis that shows that the physical degrees of freedom of linearized gravity are the symmetric, tracefree and transverse parts of $e^{a i}$ and $A_{a i}$. Although this calculation is not directly used in what follows, it is nonetheless instructive for two reasons. First, since the phase space of the linearized theory is the same as that of a triplet of Maxwell fields, a priori it is not clear that the theory represents excitations of a single spin-2 field rather than a triplet of spin-1 fields. It is therefore worthwhile to see how the presence of the vector and the scalar constraints, which have no analogs in the theory of spin-1 fields, conspire to extract precisely a single spin-2 excitation. The second reason is that the argument given here is essentially reproduced while solving the quantum constraint in section 3.3.

To extract the true degrees of freedom, it is simpler (although by no means essential, see [14]) to work in the momentum space. For this, it is convenient to introduce the standard unit basis vectors $m^{a}(k), \bar{m}^{a}(k)$ and $\hat{k}^{a}$ satisfying:

$$
m_{a}(k) k^{a}=0, m_{a}(k) m^{a}(k)=0, m_{a}(k) \bar{m}^{a}(k)=1, \quad \hat{k}^{a}=\frac{k^{a}}{|k|} .
$$

In terms of these, the metric $q_{a b}^{o}(k)$ is given by $q_{a b}^{o}=\hat{k}_{a} \hat{k}_{b}+m_{a} \bar{m}_{b}+\bar{m}_{a} m_{b}$ and the alternating tensor $\epsilon_{a b c}(k)$ will be chosen to satisfy: $\epsilon_{a b c} \hat{k}^{a} m^{b} \bar{m}^{c}=-\imath$. Let us now expand the Fourier transforms of the basic canonical variables in this basis:

$$
\begin{aligned}
A_{a b}(k)= & A^{+} m_{a} m_{b}+A^{-} \bar{m}_{a} \bar{m}_{b}+A^{1} \hat{k}_{a} m_{b}+A^{\overline{1}} \hat{k}_{a} \bar{m}_{b}+A^{2} m_{a} \hat{k}_{b} \\
& +A^{2} \bar{m}_{a} \hat{k}_{b}+A^{3} \hat{k}_{a} \hat{k}_{b}+A^{4} m_{a} \bar{m}_{b}+A^{5} \bar{m}_{a} m_{b} \\
e_{a b}(k)= & \left.e^{+} m_{a} m_{b}+e^{-} \bar{m}_{a} \bar{m}_{b}+e^{1} \hat{k}_{a} m_{b}+e^{\overline{1}} \hat{k}_{a} \bar{m}_{b}+e^{2} m_{a} \hat{k}_{b}+e^{\hat{2}} \bar{m}_{a} \hat{k}_{(} 19\right) \\
& +e^{3} \hat{k}_{a} \hat{k}_{b}+e^{4} m_{a} \bar{m}_{b}+e^{5} \bar{m}_{a} m_{b} .
\end{aligned}
$$


The scalar constraint (9) reads $\epsilon^{a b i} k_{a} A_{b i}=0$, from which we conclude $A^{4}=$ $A^{5}$. The three components of the vector constraint (8) yield, $A^{2}=A^{\overline{2}}=$ $A^{4}+A^{5}=0$. The three components of the gauge constraint 1 yield $e^{3}=0$, and gives us two relations

$$
e^{1}=\frac{\imath}{|k|} A^{1}, \quad e^{\overline{1}}=\frac{\imath}{|k|} A^{\overline{1}} .
$$

Thus, the seven constraints kill five components of the fields and give us two more relations.

We now impose seven gauge fixing conditions. As $A_{a i}$ transforms only under internal gauge transformations, it must be used to gauge fix $\mathcal{G}_{L}^{i}$. The standard Lorentz gauge condition $k^{a} A_{a i}=0$ then sets $A^{3}, A^{1}$ and $A^{\overline{1}}$ to zero, which then forces $e^{1}$ and $e^{\overline{1}}$ to vanish. Next, let us use the vector and the and scalar constraints to fix $e_{a i}$ to be symmetric. However, note that only the transverse part of the antisymmetric piece of $e_{a i}$ transforms under the canonical transformation generated by the vector constraint. We have

$$
\delta_{v} e_{[a i]}=\partial_{[i} v_{a]} .
$$

Thus, if we impose the condition

$$
\epsilon^{c a i} e_{a i}=0,
$$

only the components of this proportional to $m_{c}$ and $\bar{m}_{c}$ fix the linearized vector constraint. To fix the transverse part of this constraint we impose the trace-free condition.

$$
e_{a a}=0 .
$$

Finally, the remaining, longitudinal part, which is

$$
\epsilon^{c a i} \partial_{c} e_{a i}=0
$$

gauge fixes the scalar constraint, as one may check that

$$
\delta_{N} \epsilon^{c a i} \partial_{c} e_{a i}=-\nabla^{2} N
$$

These equations set $e^{2}=e^{\overline{2}}=e^{4}=e^{5}=0$, so we are left with the physical degrees of freedom:

$$
A_{a b}^{P h y}(k)=A^{+} m_{a} m_{b}+A^{-} \bar{m}_{a} \bar{m}_{b},
$$




$$
e_{a b}^{P h y}(k)=e^{+} m_{a} m_{b}+e^{-} \bar{m}_{a} \bar{m}_{b} .
$$

The fields with $\mathrm{a}+$ superscript correspond to the positive helicity and those with a -, to negative. Thus, in this framework, the self dual connection itself carries excitations of both helicities. This is possible because we have not made a restriction to positive frequency fields [11].

\subsection{Reality conditions}

The new canonical variables for full general relativity are somewhat unusual in that the configuration variable $A_{a i}$ is complex. To ensure that one recovers the theory of interest - real general relativity- one has therefore to impose suitable restrictions on the canonical variables. These are the reality conditions. They have to be introduced only on the initial data; they are automatically preserved under time evolution. (For a detailed discussion of why this is a viable procedure, see [4] .) In the full theory, these conditions can be expressed as [1]:

$$
\begin{aligned}
\overline{E^{a i}} & =E^{a i}, \\
A_{a}^{i}+\overline{A_{a}^{i}} & =\frac{2}{G} \Gamma_{a}^{i}(E),
\end{aligned}
$$

where $\Gamma_{a}^{i}(E)$ is the spin connection associated with the frame fields $E^{c k}$. (There exist an alternate, manifestly polynomial expression of these conditions [4]. However, it is not needed for the analysis of the linearized theory.) If we linearize these expressions, we have, in terms of the Fourier components,

$$
\begin{gathered}
\overline{e^{a b}(k)}=e^{a b}(-k), \\
A_{a}^{i}(k)+\overline{A_{a}^{i}(-k)}=\frac{2}{G} l \epsilon^{b c i}\left(k_{c} e_{(a b)}(k)-\frac{1}{2} k_{c} \delta_{a b} e(k)-\frac{1}{2} k_{a} e_{b c}(k)\right) .
\end{gathered}
$$

In the quantum theory we will only need to impose the reality conditions on the physical degrees of freedom. In the notation of the last section, these conditions are:

$$
\begin{gathered}
\overline{e^{ \pm}(k)}=e^{ \pm}(-k), \\
A^{ \pm}(k)+\overline{A^{ \pm}(-k)}=- \pm \frac{2}{G}|k| e^{ \pm}(k) .
\end{gathered}
$$


For later use, let us recast this last expression in terms of the magnetic fields. For the physical components we find

$$
B^{ \pm}(k)= \pm|k| A^{ \pm}(k)
$$

which gives us,

$$
B^{ \pm}(k)+\overline{B^{ \pm}(-k)}=-2 \frac{k^{2}}{G} e^{ \pm}(k) .
$$

This completes the summary of the phase space formulation of the linearized theory in terms of new canonical variables.

\subsection{Linearized classical loop algebra}

The loop representation in full quantum general relativity was constructed as a linear representation on the vector space of (regular) functionals on the loop space of a certain algebra of (internal) gauge invariant operators, known as the loop algebra [3]. In this paper, we want to introduce the analogous construction for the linearized theory. In this subsection, we will complete the first step by defining the appropriate classical loop variables and presenting their Poisson algebra.

The first important property of the loop variables is that they are invariant under the internal gauge transformations. The simplest loop variables which are invariant under the linearized $(U(1) \times U(1) \times U(1))$ transformations generated by $\mathcal{G}_{L}$ are

$$
t^{i}[\gamma] \equiv e^{G \oint_{\gamma} A_{a}^{i} \dot{\gamma}^{a} d s}
$$

This is an Abelian holonomy, since the connection is now Abelian. Note that the internal index has become a label on the loop variable $t^{i}[\gamma]$. Since the connection is left untouched by the canonical transformations generated by the vector and the scalar constraints, it follows immediately that these loop variables commute also with all the remaining constraints and are thus physical observables of the linearized theory.

The $t^{i}[\gamma]$ satisfy several relations, by virtue of their definitions. First, they are invariant under monotonic reparametrizations of the loops. Second, if $\alpha$ and $\beta$ are two loops that meet at a common base point, we have

$$
t^{i}[\alpha] t^{i}[\beta]=t^{i}[\alpha \circ \beta]
$$


where $\alpha \circ \beta$ is the loop obtained by combining $\alpha$ and $\beta$ (using a line segment to join them if they do not intersect). Finally, if $l$ is an open line, we have the "retracing" identity

$$
t^{i}\left[\alpha \circ l \circ l^{-1}\right]=t^{i}[\alpha]
$$

To express the dynamics of the linearized theory in terms of the loop variables, we need to write the "magnetic field" (15) as a function of the holonomy $t^{i}[\gamma]$. This can be done using a limiting procedure. For each point $x$, a unit vector $\hat{e}^{c}$ at $x$ and a real number $\delta$, we define a loop $\gamma_{x, \delta}^{c}$, which is a circle of radius $\delta$ in the plane perpendicular to $\hat{e}^{c}$ and centered at $x$. Then, as in Maxwell theory, the "magnetic field" $B^{c i}(x)$ is given by :

$$
G B^{c i}(x)=\lim \frac{1}{\pi \delta^{2}}\left(t^{i}\left[\gamma_{x, \delta}^{c}\right]-1\right)
$$

This concludes the discussion of the classical configuration loop variables. Let us now introduce the momentum loop variables. In the full theory, these momenta are "handed" loop variables, associated with oriented loops. In the linearized theory, the momentum variables are considerably simpler. From the form of the internal gauge transformation (10) generated by the linearized Gauss constraint, it is clear that $h^{a b}:=2 e^{(a b)}$ is gauge invariant. Therefore, we can just take it to be our momentum variable. Note that the origin of this simplicity is the Abelian nature of the present internal gauge group. In the full theory, by contrast, the group is non-Abelian and the construction of the momentum loop variables is more involved. Note that, since they depend only on the triads, these momenta have vanishing Poisson brackets among themselves. Thus, the only non- zero bracket between the $t^{i}[\gamma]$ and $h^{a b}(x)$ are:

$$
\left\{h^{a b}(x), t^{i}[\gamma]\right\}_{L}=-2 \imath G\left(\oint d s \delta^{3}(x, \gamma(s)) \dot{\gamma}^{(a} \delta^{b) i}\right) t^{i}[\gamma],
$$

where no sum is performed over $i$ in the right hand side. The variables $h^{a b}$ and $t^{k}[\gamma]$ will be called the linearized loop variables even though now the momentum variable is not associated with a loop. The vector space generated by them is clearly closed under the Poisson bracket. This Poisson algebra will be called the linearized loop algebra. In spite of this apparent difference between the construction of loop variables in the full and the linearized theory, it does turn out that the linearized loop algebra can be recovered from 
the loop algebra of the full theory through an appropriate limiting procedure [19].

For later use, let us decompose $h^{a b}$ in Fourier components

$$
h^{a b}(k)=\frac{1}{(2 \pi)^{\frac{3}{2}}} \int d^{3} x e^{\imath k \cdot x} h^{a b}(x) .
$$

The basic Poisson bracket of the linearized theory can now be written as:

$$
\left\{h^{a b}(k), t^{i}[\gamma]\right\}_{L}=-2 \imath G\left(F^{(a}[k, \gamma] \delta^{b) i}\right) t^{i}[\gamma]
$$

where $F^{a}[k, \gamma]$ is given by:

$$
F^{a}[k, \gamma]=\frac{1}{(2 \pi)^{\frac{3}{2}}} \oint d s e^{\imath k \cdot \gamma(s)} \dot{\gamma}^{a}(s)
$$

This quantity plays a crucial role in the description of the theory that follows; it will be called the form factor of the loop $\gamma$. The form factor is automatically transverse:

$$
k_{a} F^{a}[k, \gamma]=0,
$$

and it can be thought of as the Fourier transform of a distribution having support on the loop itself:

$$
\begin{gathered}
F^{a}[k, \gamma]=\frac{1}{(2 \pi)^{\frac{3}{2}}} \int d^{3} x e^{i k x} F^{a}[x, \gamma], \\
F^{a}[x, \gamma]=\oint d s \dot{\gamma}^{a}(s) \delta^{3}(x, \gamma(s)) .
\end{gathered}
$$

Finally, $F^{a}[k, \gamma]$ have a dual interpretation. First, if one fixes a loop $\gamma_{o}$ then $F^{a}\left[k, \gamma_{o}\right]$, regarded as a function of $k$, has the full knowledge of the spatial structure of (the holonomic equivalence class of) the loop $\gamma_{o}$. This is why, following the terminology used in particle physics, we have called $F^{a}[k, \gamma]$ a form factor. However, if one fixes a momentum vector $k_{o}$, then $F^{a}\left[k_{o}, \gamma\right]$, regarded as functions of $\gamma$, are just the logarithms of holonomies of certain connections. To see this, note first that since $F^{a}[k, \gamma]$ is transverse, it has only two components:

$$
F^{a}[k, \gamma]=F^{+}[k, \gamma] m^{a}(k)+F^{-}[k, \gamma] \bar{m}^{a}(k) .
$$


$F^{ \pm}\left[k_{o}, \gamma\right]$ is the logarithm of the holonomy of the plane wave connection with wave vector $k_{o}$ and positive (respectively, negative) helicity. This dual interpretation makes several mathematical properties of form factors transparent. We will see in the next section that these form factors play an important role in quantum theory.

\section{Quantum Theory}

This section is divided into seven parts. In the first, we introduce the loop representation for the linearized theory. In the second, we make a mathematical digression to show how the form factors introduced in section 2.4 can be used to select the appropriate class of "regular" functions on the loop space. In the third, we present the solutions to the quantum constraints and in the fourth we show how the inner product can be selected on the space of physical states using reality conditions. The fifth subsection gives the expression of the quantum Hamiltonian operator in the loop representation and provides the physical interpretation to various operators and states. A part of the construction up to this point is formal in that it contains certain divergent integrals. These are discused in the sixth subsection. The seventh shows how these divergences can be avoided by introducing suitable smearing procedures. As explained in the Introduction, we could have introduced the smearing right in the beginning of this section thereby avoiding the issue of divergences altogether. We chose not to do so only in order to simplify the presentation.

\subsection{Linearized loop representation}

The main idea of the loop representation is to construct the quantum loop algebra starting from the classical one and then find its representation using, for the carrier space, a space of functions over a loop space. (Note that in such a representation the quantum states are not functions on a configuration space. A general discussion on the loop representation can be found in [3, 4, 5). The construction of the quantum loop algebra is straightforward. It is generated by the quantum holonomy operators $\hat{t}^{i}[\gamma]$ and their "momenta" $\hat{h}^{a b}(k)$ which, for brevity, will be called the "graviton operators". The basic 
(non vanishing) commutation relation is:

$$
\left[\hat{h}^{a b}(k), \hat{t}^{i}[\gamma]\right]=2 G \hbar\left(F^{(a}[k, \gamma] \delta^{b) i}\right) \hat{t}^{i}[\gamma]
$$

where, as before, $F^{a}[k, \gamma]$ is the form factor of the loop $\gamma$. A new issue does arise, however, in the construction of the representation of this algebra. For, unlike in the loop algebra of the full theory, the holonomy operators $\hat{t}^{i}[\gamma]$ now carry an internal index. Consequently, we can no longer just repeat the procedure used in [3]. To address this issue, a new strategy is needed. In this paper, the solution we propose involves a change in the carrier space of the representation itself: in place of functions of loops, we now use functions on the space of triplets of loops. Roughly, the reason for this choice is the following. Kinematically we can think of linearized gravity in the new variables as three copies of Maxwell theory, labelled by the index $i$. The appropriate carrier space for the Maxwell loop algebra is a space of functions of (single) loops [9]. Since, in general, the appropriate space of quantum states for a composite system is the tensor product of the space of states for the single system, it is now natural to replace the loop space with the triple Cartesian product of this space with itself.

Let us begin with some definitions. By a single loop we mean a piecewise smooth closed curve $\alpha$ in $R^{3}$. A multiple loop $\eta=\left\{\alpha_{1} \cup \alpha_{2} \cup \ldots \cup \alpha_{n}\right\}$, will stand for a collection of a finite number of single loops. We use the same notation (a Greek letter) for multiple loops and for single loops (single loops being considered a special case of multiple ones). The line integral around a multiple loop $\eta$ is defined to be the sum of the line integrals around its components $\alpha_{n}$ :

$$
\oint_{\eta} f_{a} \dot{\eta}^{a} d s \equiv \sum_{n} \int_{\alpha_{n}} f_{a}\left(\dot{\alpha}_{n}\right)^{a} d s
$$

Next, let us identify two single loops $\alpha$ and $\beta$ if they have the same support and the same orientation; or, if they are related by a retracing identity $\beta=$ $\alpha \circ l \circ l^{-1}$ (see equation (38)). In addition, let us identify two multiple loops $\eta$ and $\rho$ whenever (see equation (37)

$$
\eta=\{\alpha \cup \beta \cup \gamma \cup \ldots \delta\}, \quad \rho=\{\alpha \circ \beta \cup \gamma \cup \ldots \delta\} .
$$

The space of (oriented) multiloops with these identifications will be called the $U(1)$-holonomic loop-space (or, simply loop space if there is no ambiguity), 
and denoted $\mathcal{H L}$. (This space has also ben called the non-parametric loop space by Gambini and Trias [6].) The reason for the name "holonomic loop space" is that the identifications realize the relations induced on loops by the properties of $U(1)$ holonomies. An important consequence of these identifications is that any multiloop is equivalent to a single loop. Thus, strictly speaking, it would have sufficed to work with single loops; our present description has a redundancy. However, as we will see below, many of the basic formulas are easier to express in terms of multi-loops. As emphasized and exploited by Gambini and Trias [6], $\mathcal{H L}$ has a natural group structure.

Let us next consider the space $\mathcal{H} \mathcal{L}^{3}=\mathcal{H} \mathcal{L} \times \mathcal{H L} \times \mathcal{H} \mathcal{L}$ formed by triples of multiple loops. An element of $\mathcal{H L}^{3}$ is denoted $\vec{\eta}$ :

$$
\begin{aligned}
\vec{\eta} & =\left\{\eta^{1}, \eta^{2}, \eta^{3}\right\} \\
& =\left\{\left\{\alpha_{1}^{1} \cup \alpha_{2}^{1} \cup \ldots \cup \alpha_{n_{1}}^{1}\right\},\left\{\alpha_{1}^{2} \cup \alpha_{2}^{2} \cup \ldots \cup \alpha_{n_{2}}^{2}\right\},\left\{\alpha_{1}^{3} \cup \alpha_{2}^{3} \cup \ldots \cup \alpha_{n_{3}}^{3}\right\}\right\} .
\end{aligned}
$$

The $i$-th multiple loop in the triple is denoted $\eta^{i}$. For simplicity, we will use the same term, loop, to denote single loops, multiple loops, or triplets of multiple loops; the context will make the precise meaning clear. Note also that $\mathcal{H} \mathcal{L}^{3}$ can also be seen as the space of the collections of single loops of three kind (three colors): $\eta^{1}, \eta^{2}$ and $\eta^{3}$. The space $\mathcal{H} \mathcal{L}^{3}$ is naturally equipped with an operation, the union:

$$
\vec{\eta} \cup \vec{\rho}:=\left\{\eta^{1} \cup \rho^{1}, \eta^{2} \cup \rho^{2}, \eta^{3} \cup \rho^{3}\right\} .
$$

It is convenient also to define union of a specific kind, $\cup_{i}$, of an element of $\mathcal{H L}^{3}$ with a single loop. For example, we have:

$$
\vec{\eta} \cup_{1} \alpha:=\left\{\eta^{1} \cup \alpha, \eta^{2}, \eta^{3}\right\} .
$$

With these preliminaries out of the way, we are now ready to introduce the loop representation. Th carrier space will be the space $\mathcal{S}$ of (suitably regular) functionals on $\mathcal{H} \mathcal{L}^{3}$. Thus, each $\psi \in \mathcal{S}$ has the form:

$$
\psi[\vec{\eta}]=\psi\left[\eta^{1}, \eta^{2}, \eta^{3}\right] .
$$

The regularity conditions will be specified in the next subsection. The representation map is defined as follows. The action of the holonomy operator $\hat{t}^{i}[\gamma]$ is given by :

$$
\left(\hat{t}^{i}[\alpha] \psi\right)[\vec{\eta}]:=\psi^{\prime}[\eta]=\psi\left[\vec{\eta} \cup_{i} \alpha\right] .
$$


Thus, the operator $\hat{t}^{i}[\alpha]$, operating on a state $\psi$ produces a new state $\psi^{\prime}$ whose value, on the loop $\vec{\eta}$, is the value of the old state $\psi$ on the loop $\vec{\eta} \cup_{i} \alpha$. (This operator is analogous to or translation operator in wave mechanics: $\left.(U(a) \psi)(x):=e^{\imath a \hat{p}} \psi(x)=\psi(x+a)\right)$. The second basic loop operator is the graviton operator $\hat{h}_{a b}(k)$. Its action will be given by:

$$
\left(\hat{h}^{a b}(k) \psi\right)[\vec{\eta}]=2 \hbar G F^{(a}\left[k, \eta^{b)}\right] \psi[\vec{\eta}]
$$

where $\eta^{b}$ is the $b^{\prime}$ th element of the triple of loops $\vec{\eta}$. Thus, $\hat{h}^{a b}(k)$ is in fact diagonal in the loop basis (and therefore analogous to the $\hat{x}$ operator of wave mechanics). A straightforward calculation shows that the operators $\hat{t}^{i}[\gamma]$ and $\hat{h}^{a b}$ so defined satisfy the required commutation relations; we do indeed have a representation of the quantum loop algebra. This completes the kinematics of the quantum theory.

To define the dynamics -i.e. to specify the constraint and the Hamiltonian operators- we need the operator corresponding to the the linearized magnetic field (15). By using its expression in terms of the loop observables, equation (39), we have

$$
\begin{aligned}
\hat{B}^{c i}(x) \psi[\vec{\alpha}] & \equiv \frac{1}{G} \lim _{\delta \rightarrow 0} \frac{1}{\pi \delta^{2}}\left(\hat{t}^{i}\left[\gamma_{x, \delta}^{c}\right]-1\right) \psi[\vec{\alpha}] \\
& =\frac{1}{G} \lim _{\delta \rightarrow 0} \frac{1}{\pi \delta^{2}}\left(\psi\left[\vec{\alpha} \cup\left(\gamma_{x, \delta}^{c}\right)^{i}\right]-\psi[\vec{\alpha}]\right) \\
& \equiv \frac{1}{G} \frac{\delta}{\delta \gamma^{c i}(x)} \psi[\vec{\alpha}] .
\end{aligned}
$$

In the last step we have introduced the notation $\delta / \delta \gamma^{c i}(x)$ to emphasize that the operator $\hat{B}^{a i}(x)$ can be regarded as a derivative operator in the holonomic loop- space. Note, however, that this operator is not a functional derivative. Rather, it is closely related to the operator $\Delta_{\mu \nu}(P)$ introduced by Gambini and Trias. [6] Thus, our treatment of the magnetic field operator differs from treatment one generally finds in gauge theories on loop spaces where the field operators are represented by derivative operators. This change of strategy is crucial for the case of full gravity, where the action of the limit that defines the derivative may not be well defined on diffeomorphism invariant state [3].

The linearized scalar and vector constraints can be expressed in terms of the magnetic field operator. Let us first take the Fourier transform.

$$
G \hat{B}^{c i}(k) \psi[\vec{\alpha}]=\frac{\delta}{\delta \gamma^{c i}(k)} \psi[\vec{\alpha}] \equiv \frac{1}{(2 \pi)^{\frac{3}{2}}} \int d^{3} x e^{\imath k \cdot x} \frac{\delta}{\delta \gamma^{c i}(x)} \psi[\vec{\alpha}] .
$$


The linearized constraint operators can then be expressed as:

$$
\hat{\mathcal{V}}_{a}^{L}(k)=\frac{i}{G} \epsilon_{a c i} \frac{\delta}{\delta \gamma^{c i}(k)}
$$

and

$$
\hat{\mathcal{S}}^{L}(k)=-\frac{2 i}{G} \frac{\delta}{\delta \gamma_{c}^{c}(k)}
$$

We will see later that on the space of regular loop states defined in the next subsection, the action of the magnetic field operator, and hence also of the constraint and the Hamiltonian operators, can be expressed in a simple way in terms of loop form factors introduced in section 2.4 .

\subsection{Regularity conditions on loop states}

We now complete the introduction of the linearized loop representation by specifying the regularity conditions that need to be imposed on the loop functionals $\psi(\vec{\alpha})$.

For this purpose, we will use the loop form factors $F^{a}[k, \gamma]$. Note first that $F^{a}[k, \gamma]$ are well-defined on the $U(1)$-holonomic loop space $\mathcal{H} \mathcal{L}$ because two equivalent loops have the same form factors. (Recall that the form factors themselves can be regarded as $U(1)$ holonomies of certain connections.) Since the Fourier transform of the form factor is a distribution with support on the holonomic-loop itself, it is clear that, as a function of $k$, the form factor of a given holonomic loop $\eta$ uniquely determines $\eta$. Thus, seen as functions of $k$, the form factors serve as good labels for holonomic-loops. They cannot be regarded as coordinates in the technical sense because the space of form factors itself does not form a linear space; if $F^{a}\left[k, \gamma^{i}\right]$ is the form factor of $\gamma^{i}$, in general $\lambda F^{a}\left[k, \gamma^{i}\right]$ will not be a form factor of any loop. Nonetheless, we have the following interesting mathematical structure. Consider the space $\mathcal{F}^{3}:=\mathcal{F} \times \mathcal{F} \times \mathcal{F}$, where $\mathcal{F}$ is the space of vector fields $F^{a}(k)$ in the momentum space satisfying $\overline{F^{a}(k)}=F^{a}(-k)$ and $F^{a}(k) k_{a}=0$. The first condition ensures that $F^{a}(k)$ is the Fourier transform of a real vector field $F^{a}(x)$ while the second ensures that $F^{a}(x)$ is divergence free. (A more precise definition of this function space will be given later.) Form factors $F^{a}\left[k, \gamma^{i}\right]$ can be now regarded as 1-1 mappings from the loop space $\mathcal{H} \mathcal{L}^{3}$ into the space $\mathcal{F}^{3}$. 
Therefore, any functional $\phi\left(F^{a i}(k)\right)$ on $\mathcal{F} \times \mathcal{F} \times \mathcal{F}$ determines uniquely a functional on loop space via

$$
\psi[\vec{\gamma}]=\left.\phi\left[F^{a i}(k)\right]\right|_{F^{a i}(k)=F^{a}\left(k, \gamma^{i}\right)}
$$

and any loop functional can be obtained in this way. Thus, the space of the functionals $\phi$ on $\mathcal{F}^{3}$ is mapped linearly onto the space of the loop functionals. We now exploit this fact to specify the regularity conditions on the loop functionals $\psi(\vec{\gamma})$ in terms of those on the functionals $\phi\left(F^{a i}(k)\right)$.

A loop functional $\psi(\gamma)$ will be said to be regular -and thus belong to the carrier space of the representation- if and only if it is obtained via (61) from a functional $\phi$ which admits a convergent Taylor expansion on the space $\mathcal{F}^{3}$. Thus, regular loop states arise from analytic functionals on $\mathcal{F}^{3}$. Let us give a few examples. A linear functional on $\mathcal{F}^{3}$ is of the type $\phi\left(F^{a i}(k)\right)=\int d^{3} k C_{a i}(k) F^{a i}(k)$, while a $n$-th order functional is of the type $\int d^{3} k_{1} \ldots \int d^{3} k_{n} C_{a_{1} i_{1} \ldots a_{n} i_{n}}\left(k_{1}, \ldots k_{n}\right) F^{a_{1} i_{1}}\left(k_{1}\right) \ldots . F^{a_{n} i_{n}}\left(k_{n}\right)$. Any regular loop state $\psi(\gamma)$ is obtained via (61) from a (possibly infinite, convergent) linear combination of these n-nomials on $\mathcal{F}^{3}$. Note that the linear functionals have six degrees of freedom for each momentum value $k$, since it is only the transverse part of $C_{a i}(k)$ in the index $a$ that contributes to the integral. We will see that the imposition of the (four) scalar and vector constraints reduce the degrees of freedom precisely to two. These will correspond to the two degrees of freedom of the graviton discussed in section 2.2. (A key feature of the loop representation is that the quantum Gauss constraint does not have to be imposed; it is automatically satisfied. This is a very general property. It holds also in full general relativity, as well as in the Maxwell and Yang-Mills theories.)

Note that since the map from $\mathcal{H} \mathcal{L}^{3}$ to $\mathcal{F}^{3}$ is into and not onto (i.e. injective and not surjective), the linear mapping (61) has a non-trivial kernel. In various calculations, we will need to express the action of the loop operators on states $\psi(\vec{\gamma})$ using form factors $F^{a i}(k)$. Therefore, it is important to ask if the restriction of the map (61) to polynomials $\phi\left(F^{a i}(k)\right)$ is faithful: If it is not, we will not be easily able to go back and forth between operators on loop states $\psi(\vec{\gamma})$ and those on polynomial functionals $\phi\left(F^{a i}(k)\right)$ on $\mathcal{F}^{3}$. Fortunately, it turns out that the kernel of the restricted map is in fact trivial. We can therefore pass freely between the regular loop states $\psi(\vec{\gamma})$ and the polynomial functionals $\phi\left(F^{a i}(k)\right)$ on $\mathcal{F}^{3}$ from which they arise. 
To prove this assertion, we proceed in two steps. First we show that distinct n-nomials of the same degree give rise to distinct loop functionals. Let us begin with degree one, i.e., linear functionals. The problem then reduces to showing that, if for every loop $\alpha$ in $\mathcal{H L}$ we have

$$
\int d^{3} k \quad c_{a}(k) F^{a}[k, \alpha]=0,
$$

then for every $F^{a}(k)$ in $\mathcal{F}$, we must have:

$$
\int d^{3} k \quad c_{a}(k) F^{a}(k)=0 .
$$

This is easy to establish. Equation (62) implies:

$$
\int d^{3} k c_{a}(k) \oint d s e^{\imath k \alpha(s)} \dot{\alpha}^{a}(s)=\oint d s \dot{\alpha}^{a}(s) c_{a}(\alpha(s))=\oint_{\alpha} d S^{a} c_{a}=0,
$$

where $c_{a}(x)$ is the Fourier transform of $c_{a}(k)$. Since its line integral on any loop is zero, $c_{a}(x)$ is curl free, whence $c_{a}(k)=k_{a} c(k)$. Inserting this in (63), we obtain the required result because every $F^{a}(k)$ in $\mathcal{F}$ is transverse. Let us next consider a quadratic functional on $\mathcal{F}$. Let us suppose that the restriction of this functional to the $F^{a}(k)$ that arise from form factors of arbitrary loops vanishes. Then, we have, for all single loops $\alpha$ and $\beta$ :

$$
\int d^{3} k_{1} \int d^{3} k_{2} c_{a b}\left(k_{1}, k_{2}\right) F^{a}\left[k_{1}, \alpha\right] F^{b}\left[k_{2}, \alpha\right]=0,
$$

and, considering a multiple loop formed by these two single loops, we also have

$$
\int d^{3} k_{1} \int d^{3} k_{2} c_{a b}\left(k_{1}, k_{2}\right) F^{a}\left[k_{1}, \alpha \cup \beta\right] F^{b}\left[k_{2}, \alpha \cup \beta\right]=0 .
$$

Using the fact that the form factor of the union of two loops is the sum of the form factors, we conclude, from the last two equations,

$$
\int d^{3} k_{1} \int d^{3} k_{2} c_{a b}\left(k_{1}, k_{2}\right) F^{a}\left[k_{1}, \alpha\right] F^{b}\left[k_{2}, \beta\right]=0 .
$$

Now we can repeat the argument used in the linear case, and conclude that $c_{a b}\left(k_{1}, k_{2}\right)=\left(K_{1}\right)_{a}\left(k_{2}\right)_{b} c\left(k_{1}, k_{2}\right)$, whence the quadratic functionals have to vanish everywhere on $\mathcal{F}$. It is clear that the discussion can be repeated for an arbitrary n-nomial: If the restriction of an n-nomial on $\mathcal{F}$ to the image of 
the loop space $\mathcal{H} \mathcal{L}$ vanishes, then the n-nomial must vanish everywhere on $\mathcal{F}$. Finally, it is straightforward to replace in this result $\mathcal{F}$ by $\mathcal{F}^{3}$ and $\mathcal{H} \mathcal{L}$ by $\mathcal{H} \mathcal{L}^{3}$.

The second step in the argument uses this fact to establish that the same result holds for an arbitrary linear combination of the n-nomials, i.e., an arbitrary polynomial. That is, we have to show that the restrictions to the image of $\mathcal{H} \mathcal{L}$ of functions which are n-nomials of different oder are necessarily linearly independent. To carry out this step, we introduce the following linear operator on the space of loop states:

$$
\hat{P} \psi[\vec{\alpha}]:=\psi[\vec{\alpha} \cup \vec{\alpha}] .
$$

It is straightforward to check that every n-nomial functional $\phi\left(F^{a i}(k)\right)$ of degree $n$ has the property that if $\psi\left(\alpha^{i}\right)=\phi\left(F^{a}\left(k, \alpha^{i}\right)\right)$, then:

$$
\hat{P} \psi(\vec{\alpha})=2^{n} \psi(\vec{\alpha}) .
$$

Consequently, the restrictions to the image of $\mathcal{H} \mathcal{L}^{3}$ of n-nomial with different degree belong to the eigenspaces of $\hat{P}$ with different eigenvalues. Therefore they cannot be linearly dependent. (To see this, note that, since the eigenvalues are all real, one can introduce an inner-product with respect to which the operator $\hat{P}$ is Hermitian and hence these distinct eigenspaces are mutually orthogonal. In fact, the operator is closely related to the number operator in quantum theory.)

Thus, we have shown that the space of regular functionals on the loop space is indeed isomorphic with the space of polynomials on a linear space, $\mathcal{F}^{3}$. This fact will be used repeatedly in the calculations that follow. Finally, note that we have purposely left the precise definition of the function space $\mathcal{F}$ open at this stage. The appropriate function space will be singled out by physical considerations involving the inner product and the Hamiltonian in section 3.7 .

\subsection{Solutions to the constraints: physical states}

Let us now impose the linearized scalar and vector constraints on the (regular) loop states. That is, let us find the subspace $\mathcal{S}_{P h y} \subset \mathcal{S}$ annihilated by the operators $\hat{\mathcal{V}}_{a}^{L}$ and $\hat{\mathcal{S}}^{L}$ defined in (59) and (60). 
To carry out this task, it is convenient to first evaluate the action of the magnetic field operator $\hat{B}^{a i}(k)$ on regular loop states. If $\psi(\vec{\alpha})=\phi\left(F^{a}\left(k, \alpha^{i}\right)\right)$, then using the definition (58) of the operator $\delta / \delta \gamma^{a i}(k)$, the expression (58) of the magnetic field operator simplifies to:

$$
G \hat{B}^{c i}(k) \cdot \psi[\vec{\alpha}]=\int d^{3} p \frac{\delta \phi}{\delta F^{b j}(k)} \frac{\delta F^{b}\left(p, \gamma^{j}\right)}{\delta \gamma^{a i}(k)} .
$$

Now, from the definition (43) of form factors, it is straightforward to evaluate the second term. We have:

$$
\frac{\delta}{\delta \gamma^{a i}(k)} F^{b}\left[p, \gamma^{j}\right]=\imath \delta^{3}(k+p) \epsilon^{b a c} k_{c} \delta^{i j},
$$

Substituting this result in the above expression of the magnetic field operator, we obtain:

$$
G \hat{B}^{c i}(k) \cdot \psi[\vec{\alpha}]=i C^{a i}(-k) \epsilon_{a}^{c d} k_{d},
$$

where we have set

$$
C_{a i}(k):=\frac{\delta \phi}{\delta F^{a i}(k)} .
$$

Equation (72) now implies the following necessary and sufficient conditions for $\psi[\vec{\alpha}]$ to be a physical state:

$$
\hat{\mathcal{S}}^{L} \psi[\vec{\alpha}]=0 \text { iff } \epsilon^{a i c} k_{c} C_{a i}(k)=0
$$

and

$$
\hat{\mathcal{V}}_{e}^{L} \psi[\vec{\alpha}]=0 \quad \text { iff } \quad k^{c} C_{e c}(k)-k_{e} C_{a}^{a}(k)=0 .
$$

Note, furthermore, that, because $F^{a i}(k)$ in $\mathcal{F}^{3}$ are all transverse, the functional derivative of $\phi$ with respect to them is determined only up to an additive term of the type $k_{a} v_{i}(k)$. Using this freedom, without any loss of generality, we can restrict ourselves to those $C_{a i}(k)$ which satisfy:

$$
k^{a} C_{a i}(k)=0 .
$$

We now have seven equations for the nine components of the $C_{a i}(k)$. The solutions have two degrees of freedom and, not surprisingly, are symmetric, tracefree transverse fields:

$$
C_{a b}(k)=A^{+} m_{a}(k) m_{b}(k)+A^{-} \bar{m}_{a}(k) \bar{m}_{b}(k) .
$$


It follows that the general solutions are those that are functions only of the tracefree transverse components of the $F^{a}\left[k, \alpha^{i}\right]$, which are

$$
\begin{aligned}
& F^{+}[k, \vec{\alpha}] \equiv \bar{m}_{a} \bar{m}_{i} F^{a}\left[k, \alpha^{i}\right], \\
& F^{-}[k, \vec{\alpha}] \equiv m_{a} m_{i} F^{a}\left[k, \alpha^{i}\right] .
\end{aligned}
$$

That is, the general solution to the quantum constraints has the form

$$
\psi[\vec{\alpha}]=\phi\left[F^{+}[k, \vec{\alpha}], F^{-}[k, \vec{\alpha}]\right] .
$$

The space spanned by these functions will be denoted $\mathcal{S}_{P h y}$.

Finally, one can repeat the arguments presented in section 3.2 to show that there is a 1-1 correspondence between physical states $\psi[\vec{\alpha}]$ and (analytic) functionals $\phi$ on $\mathcal{F}_{P h y}^{3}:=\mathcal{F}^{+} \oplus \mathcal{F}^{-}$, where $\mathcal{F}^{ \pm}$are spanned by functions $F^{ \pm}(k)$ given by: $F^{+}(k)=F^{a i}(k) \bar{m}_{a}(k) \bar{m}_{i}(k)$ and $F^{-}(k)=F^{a i}(k) m_{a}(k) m_{i}(k)$. The spaces $\mathcal{F}^{ \pm}$can be defined in their own right (without reference to $\mathcal{F}^{3}$ ) as the vector spaces of functions $F^{ \pm}(k)$ on the momentum space with spin weight \pm 2 which satisfy the reality conditions $\overline{F^{ \pm}(k)}=F^{ \pm}(-k)$. Thus, just as we had, in section 3.2, a representation of the regular loop states by functionals on a linear space $\mathcal{F}^{3}$, we now have a representation of the physical loop states by functionals on a linear space $\mathcal{F}_{P h y}^{3}$. We will use this representation to simplify the expressions of the physical operators. The physical operators -i.e. loop operators which leave the physical space $\mathcal{S}_{P h y}$ invariant- are easy

to identify: they are $\hat{h}^{ \pm}(k)$ and $\hat{B}^{ \pm}(k)$, obtained by contracting $\hat{h}^{a b}(k)$ and $\hat{B}^{a i}(k)$ with the vectors $m^{c}(k)$ and $\bar{m}^{c}(k)$ in the obvious way. Their action on physical states can now be expressed as follows. For all $\psi[\vec{\alpha}]$ in $\mathcal{S}_{P h y}$ with $\psi[\vec{\alpha}]=\phi\left[F^{ \pm}(k, \vec{\alpha})\right]$,

$$
\begin{gathered}
\hat{h}^{ \pm}(k) \cdot \psi[\vec{\alpha}]=2 \hbar G F^{ \pm}(k, \vec{\alpha}) \quad \psi[\vec{\alpha}] \\
\hat{B}^{ \pm}(k) \cdot \psi[\vec{\alpha}]= \pm\left.\frac{|k|}{G}\left[\frac{\delta}{\delta F^{ \pm}(-k)} \cdot \phi\left(F^{ \pm}(k)\right)\right]\right|_{F^{ \pm}(k)=F^{ \pm}(k, \vec{\alpha})}
\end{gathered}
$$

\subsection{Physical inner product}

Up to this point, the space of states has the structure of only a complex vector space; it is not equipped with an inner product. The idea of postponing 
the introduction of the inner product until after one has solved the quantum constraints and thus extracted the physical states is rather common in the literature. Indeed, in most examples of constrained systems (even with just a finite number of degrees of freedom) the inner product that may seem "natural" prior to the imposition of constraints has little physical relevance because typically the physical states have infinite norms with respect to this inner product. However, in most treatments, once the physical states are singled out, the inner product is first introduced by making an appeal to a symmetry group -the Poincaré group in Minkowskian field theories- and physical observables are then taken to be the self-adjoint operators on the resulting Hilbert space. In the present quantization program, the strategy is somewhat different [17, 3, 4, 5]. We first solve the constraints, then introduce linear operators on the resulting vector space that correspond to real classical observables and in the final step seek a Hermitian inner product that makes these operators self-adjoint. Thus, the requirement that real classical observables should become self-adjoint operators is now regarded as a restriction on the choice of the inner product. Consequently, the program does not rely on the Poincaré invariance which has no obvious analog in quantum gravity. Furthermore, this strategy allows us to begin with complex canonical variables to describe the real gravitational field. In the classical theory, one recovers the real theory by imposing the reality conditions on the canonical variables. In the quantum theory, these conditions dictate Hermitian adjointness relations and therefore also the choice of the inner product. Thus, our program is an extension of the Dirac method of quantizing constrained systems in that it enables us to use more general canonical variables - such as the real triads but complex complex connections- and, at the same time, contains a concrete proposal to select the required inner product. (For further details on the general program, see [1].)

Let us return to linearized gravity. In this subsection, we will show that the reality conditions do indeed suffice to pick out an inner product on the space $\mathcal{S}_{P h y}$ of physical states. Moreover, eventhough no explicit appeal is made to Poincaré invariance, the inner product we find is the correct one: the final theory is completely equivalent to the theory of gravitons obtained via standard methods. The same procedure has also been shown to work in a variety of systems, such as the quantum Maxwell theory [9], $2+1$ gravity [0], and certain model systems (with a finite number of degrees of freedom) that 
mimic various features of general relativity [8]. We suspect that there is a general theorem which captures the idea that, for a wide class of constrained systems, the physical reality conditions essentially suffice to determine the inner product on the space of physical states.

We begin by positing a general form for an inner product on $\mathcal{S}_{P h y}$. The idea is to represent the regular loop states $\psi(\vec{\gamma})$ by the functionals $\phi\left(F^{ \pm}(k)\right)$ from which they arise, make the ansatz

$$
<\psi \mid \psi^{\prime}>:=\int \prod_{k, \pm} d F^{ \pm}(k) e^{-T\left[F^{ \pm}(k)\right]} \overline{\phi\left(F^{ \pm}\right)} \phi^{\prime}\left(F^{ \pm}\right)
$$

and then determine the "measure" $\exp -T\left[F^{ \pm}(k)\right]$ on $\mathcal{F}_{\text {Phy }}^{3}$ by imposing the reality conditions. Recall that $F^{ \pm}(k)$ satisfy the condition:

$$
\overline{F^{ \pm}(k, \vec{\alpha})}=F^{ \pm}(-k, \vec{\alpha})
$$

Hence, the inner product is (formally) Hermitian if and only if $T\left[F^{ \pm}(k)\right]$ is real-valued. To determine its functional form, let us now impose the reality conditions. The property (84) of $F^{ \pm}(k)$ implies that the first of the two reality conditions, (32), is automatically satisfied. The second, (35), holds if and only if

$$
<\psi\left|\left(\hat{B}^{ \pm}(k)\right)^{\dagger}\right| \chi>\equiv \overline{<\chi\left|\hat{B}^{ \pm}(k)\right| \psi>}=<\psi\left|-\hat{B}^{ \pm}(-k)-\frac{k^{2}}{G} \hat{h}^{ \pm}(-k)\right| \chi>.
$$

Using the explicit action of the operators $(81,82) \hat{h}^{ \pm}(k)$ and $\hat{B}^{ \pm}(k)$ on physical states, we conclude that the necessary and sufficient condition for the reality conditions to hold is:

$$
\frac{\delta T}{\delta F^{ \pm}(-k)}=\mp 2|k| \hbar G F^{ \pm}(k)
$$

The functional $T$ is determined uniquely (up to an additive constant) by (86):

$$
T=\hbar G \int d^{3} k|k|\left(\left|F^{-}(k)\right|^{2}-\left|F^{+}(k)\right|^{2}\right)
$$

Thus, the final form of the inner product is:

$$
<\psi \mid \psi^{\prime}>=\int \prod_{k, \pm} d F^{ \pm}(k) e^{\hbar G \int d^{3} k|k|\left(\left|F^{+}(k)\right|^{2}-\left|F^{-}(k)\right|^{2}\right)} \overline{\phi\left(F^{ \pm}\right)} \phi^{\prime}\left(F^{ \pm}\right)
$$


Let us analyze the space of normalizable physical states. Its structure is most transparent when expressed in terms of functionals $\phi\left(F^{ \pm}(k)\right)$ on $\mathcal{F}_{\text {Phy }}^{3}$. On the negative helicity sector, the inner product (88) is given by just a Gaussian integral. All polynomials in $F^{-}(k)$ are normalizable. The functionals that depend on $F^{+}(k)$ on the other hand will not be normalizable unless they are exponentially damped. Thus, the normalizable states are of the form $\phi\left(F^{ \pm}(k)\right)=P\left(F^{ \pm}(k)\right) \exp -\hbar G \int d^{3} k|k|\left|F^{+}(k)\right|^{2}$, where $P$ is a polynomial. The Hilbert space of physical states will contain more general states; as is usual in field theory, the Cauchy completion considerably expands this space of polynomials. Finally, we note that the description is again asymmetric between the two helicities. The detailed discussion of the origin and meaning of this asymmetry can be found in 4 .

\subsection{Quantum Hamiltonian and the graviton states}

The classical Hamiltonian (16) in terms of the physical degrees of freedom is

$$
\begin{aligned}
H=-\frac{1}{2} \int d^{3} k \quad & {\left[\left(h^{+}(-k)+\frac{G}{k^{2}} B^{+}(-k)\right) B^{+}(k)\right.} \\
& \left.+\left(h^{-}(-k)+\frac{G}{k^{2}} B^{-}(-k)\right) B^{-}(k)\right] .
\end{aligned}
$$

The quantum Hamiltonian operator on the physical states is therefore obtained just by replacing the classical fields $h^{ \pm}(k)$ and $B^{ \pm}(k)$ by the corresponding operators with an appropriate factor ordering. Under the inner product defined in the previous subsection, we have:

$$
-\left(\hat{h}^{ \pm}(-k)+\frac{G}{k^{2}} \hat{B}^{ \pm}(-k)\right)^{\dagger}=\frac{G}{k^{2}} \hat{B}^{ \pm}(k) .
$$

The form of the Hamiltonian and these Hermitian adjoint relations suggest that appropriate multiples of the operators $\hat{B}^{ \pm}(k)$ should be identified with the creation or the annihilation operators of gravitons. The precise multiple is determined by the commutation relations and dimensional considerations. The correct identifications turn out to be:

$$
a_{+}(k)=-\sqrt{\frac{|k|}{2 \hbar G}}\left[\hat{h}^{+}(-k)+\frac{G}{|k|^{2}} \hat{B}^{+}(-k)\right]
$$




$$
\begin{aligned}
\left(a_{+}(k)\right)^{\dagger} & =\sqrt{\frac{G}{2 \hbar|k|^{3}}} \hat{B}^{+}(k), \\
\left(a_{-}(k)\right)^{\dagger} & =-\sqrt{\frac{|k|}{2 \hbar G}}\left[2 \hat{h}^{-}(-k)+\frac{G}{|k|^{2}} \hat{B}^{-}(-k)\right] \\
a_{-}(k) & =\sqrt{\frac{G}{2 \hbar|k|^{3}}} \hat{B}^{ \pm}(k) .
\end{aligned}
$$

With this assignment of the roles to the operators, the creators and the annihilators have the correct physical dimensions, (length) ${ }^{-\frac{3}{2}}$, and satisfy the correct commutation relations; the only non-vanishing bracket is

$$
\left[a_{ \pm}(p),\left(a_{ \pm}(k)\right)^{\dagger}\right]=\delta^{3}(k-p) \text {. }
$$

Furthermore, the (normal ordered) Hamiltonian operator can now be expressed as:

$$
H=\int d^{3} k \hbar|k|\left[\left(a_{+}(k)\right)^{\dagger} a_{+}(k)+\left(a_{-}(k)\right)^{\dagger} a_{-}(k)\right] .
$$

The asymmetry in the identification of creators and annihilators for the two helicities arises because there is a difference in sign in the commutation relations between $\hat{h}^{ \pm}(k)$ and $\hat{B}^{ \pm}(k)$ which in turn arises because of our preference for self dual connections over the anti-self dual ones.

Let us examine the action of these operators on physical states. The vacuum $\psi_{o}(\vec{\gamma})$ is annihilated by the operators $a_{ \pm}(k)$. Using the expressions (81,82) of the operators $\hat{h}^{ \pm}(k)$ and $\hat{B}^{ \pm}(k)$, we have:

$$
a_{-}(k) \psi_{o}(\vec{\gamma})=\sqrt{\frac{1}{2 \hbar G|k|^{3}}} \frac{\delta}{\delta F^{-}(-k)} \phi_{o}\left(F^{ \pm}\right)=0
$$

and

$$
a_{+}(k) \psi_{o}(\vec{\gamma})=-\left(\sqrt{\frac{|k|}{2 \hbar G}}\left[|k|^{-1} \frac{\delta}{\delta F^{+}(k)}+2 \hbar G F^{+}(-k)\right]\right) \psi_{0}\left(F^{ \pm}\right)=0 .
$$

The solutions to these equations is:

$$
\psi_{o}(\vec{\gamma})=e^{-\hbar G \int d^{3} k|k|\left|F^{+}[k, \vec{\gamma}]\right|^{2}} .
$$


Note that the exponential damping with respect to the positive helicity states is precisely of the form needed by the form of the inner product (88). The expression of the vacuum state in this representation is thus chirally asymmetric. Again, this feature arises because of our asymmetric choice of basic variables: physically, the holonomy operators are tied with self dual connections. Had we worked with positive frequency connections rather than self dual, the vacuum state would have been the unit functional for both helicities. The situation is completely analogous in the self-dual loop representation of the Maxwell theory as well and is not intrinsic to gravity.

The graviton states can now be constructed by acting with the creation operators (92) and (93) on this vacuum. For example, the one graviton states are:

$$
\psi_{(p,+)}(\vec{\gamma})=\left(a_{+}(p)\right)^{\dagger} \psi_{o}(\vec{\gamma})=-\sqrt{2 \hbar G|p|} F^{+}(p) e^{-\hbar G \int d^{3} k\left|F^{+}(k)\right|^{2}}
$$

and

$$
\psi_{(p,-)}(\vec{\gamma})=\left(a_{-}(p)\right)^{\dagger} \psi_{o}(\vec{\gamma})=\sqrt{2 \hbar G|p|} F^{-}(p)^{-\hbar G \int d^{3} k|k|\left|F^{+}(k)\right|^{2}} .
$$

By superposing these pure momentum graviton states, one can construct wave packets. In terms of loops, a general one-graviton state $f_{a j}(p)=$ $f^{+}(p) m_{a}(p) m_{j}(p)+f^{-} \bar{m}_{a}(p) \bar{m}_{j}$ in the Fock space can now be expressed as:

$$
\psi[\vec{\gamma}]=\left(\sum_{j} \oint_{\alpha^{j}} f_{j a} \dot{\alpha}_{j}^{a} d s\right) \psi_{o}\left[\gamma^{i}\right]
$$

where $f_{j a}(x)$ is the Fourier transform of $f^{+}(p) m_{j}(p) m_{a}(p)-f^{-}(p) \bar{m}_{j}(p) \bar{m}_{a}(p)$. More generally, the helicity plus, m-graviton states are expressed by nnomials in $F^{+}$of order $\mathrm{m}$, while the helicity minus, m-graviton states are expressed by Hermite polynomials in $F^{-}$of order n (times the vacuum). Thus, these states have the correct functional dependence on the form factors to be formally normalizable.

\subsection{Divergences in the ground state wave functional}

We have presented a construction of the Fock space of free gravitons, together with the Hamiltonian (89), inner product (88), an algebra of creation and 
annihilation operators (91,92,93, 94), and explicit expressions for the ground state (99) and graviton states. However, certain steps in this construction are only formal. As we are about to show, the momentum integral in the expression of the ground state $\psi_{o}(\vec{\gamma})$ diverges. Therefore, regarded as a functional of loops, the ground state -and hence also any n-graviton state- wave functional vanishes on all but the trivial loop!

The origin of this problem is the following. From a physical point of view, the problem is simply that the form factor of any loop, seen as a one-particle wave-function, corresponds to a non-normalizable (improper) state. More precisely, consider the sub-space $\mathcal{F}_{o}^{3}$ of $\mathcal{F}_{\text {Phy }}^{3}$ spanned by functions $F^{ \pm}(k)$ on the momentum space for which $\int d^{3} k|k|\left|F^{ \pm}(k)\right|^{2}$ is finite. The vacuum state $\phi_{o}\left(F^{ \pm}(k)\right.$ ) (as well as any $n$ graviton state) is a non-zero, well-defined functional on this space. Why is it then that $\psi_{o}(\vec{\gamma})$ vanishes? The reason is that the form factors $F^{a i}(k, \vec{\gamma})$ are such that $\int d^{3} k|k|\left|F^{ \pm}(k, \vec{\gamma})\right|^{2}$ diverges for all (non-trivial) loops $\vec{\gamma}$. Therefore, the image within $\mathcal{F}_{\text {Phy }}^{3}$ of the loop space $\mathcal{H L}^{3}$ has zero intersection with $\mathcal{F}_{o}^{3}$. This is why the n-graviton states which, strictly speaking, have support only on the subspace $\mathcal{F}_{o}^{3}$ of $\mathcal{F}^{3}$ get pulled back to the zero functional on the loop space. Thus, the problem has to do with the precise choice of the function space $\mathcal{F}$ which we left open. To fix this choice in an appropriate fashion, we will need to thicken out the loops and use the corresponding "smeared out" form factors to map the loop space $\mathcal{H L}^{3}$ to $\mathcal{F}^{3}$. That is, to resolve the problem, we have to sharpen the regularity conditions on loop states. We will introduce the required smearing in section 3.7. Once this is done, we will be able to represent the n-graviton states as functionals on smeared out loops in an unambiguous way. Furthermore, the overall structure of the theory after smearing will remain essentially the same as it has been so far.

In this sub-section, however, we will continue to work with the unsmeared loops, examine more closely the ground state wave functional obtained above and explore the nature of the divergent integrals involved.

Using the definition of the $F^{ \pm}(k, \alpha)$ in terms of the $m(k)$ and $\bar{m}(k)$ the ground state wave-function (99) may be written

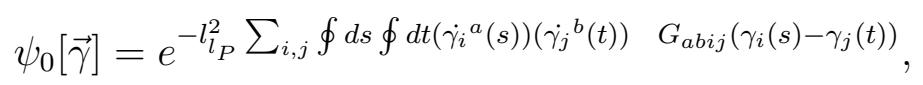


where $l_{P}=\hbar G$ is the Planck length and

$$
G_{a b i j}(x) \equiv \int \frac{d^{3} k}{(2 \pi)^{3}}|k| e^{\imath k \cdot x}\left[\delta_{i j}-\frac{k_{i} k_{j}}{k^{2}}-\imath \epsilon_{i j l} \frac{k^{l}}{|k|}\right]\left[\delta_{a b}-\imath \epsilon_{a b c} \frac{k^{c}}{|k|}\right] .
$$

We have used the identity

$$
m_{i}(k) \bar{m}_{j}(k)=\frac{1}{2}\left[\delta_{i j}-\frac{k_{i} k_{j}}{k^{2}}-\imath \epsilon_{i j l} \frac{k^{l}}{|k|}\right],
$$

and the fact that the $F^{a}[k, \gamma]$ are transverse. It is straightforward to do the momentum integrals, after which we find

$$
\begin{aligned}
G_{a b i j}(x)= & -\frac{\delta_{i j} \delta_{a b}}{|x|^{4}}+2 \frac{\delta_{a b} x^{i} x^{j}}{|x|^{6}} \\
& -\frac{1}{4}\left[\delta_{a b} \epsilon_{i j k}+\delta_{i j} \epsilon_{a b c}\right] \frac{\partial}{\partial x^{k}} \delta^{3}(x) \\
& +\frac{3}{32 \pi^{3}} \epsilon_{a b c}\left[5 \frac{x^{i} x^{j} x^{c}}{|x|^{7}}-\frac{\delta_{i j} x^{c}+\delta_{c j} x^{i}+\delta_{c i} x^{j}}{|x|^{5}}\right] \\
& +\frac{\epsilon_{i j k} \epsilon_{a b c}}{2|x|^{4}}\left[\delta_{c k}-\frac{4 x^{c} x^{k}}{|x|^{2}}\right] .
\end{aligned}
$$

Now, it is easy to see that the two integrals in the definition of the vacuum state are divergent for every non-trivial loop. Note that the divergences occur whenever $\left|\gamma_{i}(s)-\gamma_{j}(t)\right|=0$, which means either at an intersection or in the diagonal terms in the sum $\sum_{i j}$.

To clarify the nature of these divergences, let us consider the value of the ground state wave-function on a particular loop. We evaluate the integrals for non-intersecting loops, so that the divergences are only in the diagonal terms. We note that many of the possibly divergent terms can be set equal to zero if we choose the loops to be transverse, by which we mean that the $i^{\prime} t h$ loop always runs in a plane perpendicular to the $i^{\prime} t h$ direction. We then have, up to finite terms,

$$
\begin{gathered}
\psi_{0}[\vec{\gamma}]=e^{\sum_{i} W\left[\gamma_{i}\right]} \\
W[\gamma]=l_{P}^{2} \oint d s \oint d t \frac{\dot{\gamma}^{a}(s) \dot{\gamma}^{b}(t) \delta_{a b}}{|\gamma(s)-\gamma(t)|^{4}}
\end{gathered}
$$


We expand $\gamma^{a}(t)=\gamma^{a}(s)+(t-s) \dot{\gamma}^{a}(s)+\ldots$ and, for convenience, choose a parametrization such that $\left|\dot{\gamma}^{a}\right|=1$ (so that $\dot{\gamma} \cdot \ddot{\gamma}=0$ ). We find

$$
W[\gamma]=-l_{l_{P}}^{2}\left[\oint_{0}^{L} d s \oint_{0}^{L} d t \frac{1}{(t-s)^{4}}-\oint_{0}^{L} d s|\ddot{\gamma}(s) \cdot \ddot{\gamma}(s)| \oint_{0}^{L} d t \frac{1}{2(t-s)^{2}}+\ldots\right]
$$

We can draw two conclusions from this expression. First, the leading divergence is independent of the loop. Were this the only divergence (as is the case in Maxwell theory [9]) then the divergence could be removed by a wave-function renormalization of the form,

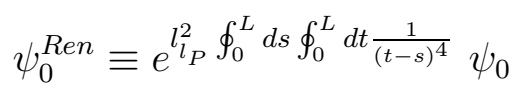

(defined in the context of an appropriate regularization procedure.) However, there are non-leading divergences which depend on the loop, and, in particular, on the averages of higher derivatives of the loop taken around the loop. Indeed, we see that even if wave-function renormalization is done, the renormalized ground state will still vanish on all loops except the straight lines.

The difference between the case of Maxwell theory and linearized gravity may be traced directly to the dimensional nature of the gravitational constant. Because of the presence of $\hbar G$, the momentum integrals are divergent like $l_{l_{P}}^{2} / \epsilon^{2}$, where $\epsilon$ is any short distance cutoff. In the Maxwell case, where there is no dimensional constant, the corresponding expression is only logarithmically divergent, so that the only divergences are loop independent.

\subsection{Averaging}

In this section, we construct a modified version of the representation defined above in which no divergent quantity appears. (Further details can be found in [9].)

To motivate the modification needed, let us first make a brief detour to note that we do already have a well-defined representation of the algebra of the the graviton operators $\hat{h}^{ \pm}(k)$ and the magnetic field operators $\hat{B}^{ \pm}(k)$ in which the states are represented by functionals $\phi\left(F^{ \pm}(k)\right)$ on the space $\mathcal{F}_{o}^{3}$. (Recall that this is the space spanned by $F^{ \pm}(k)$ for which $\int d^{3} k|k|\left|F^{ \pm}(k)\right|^{2}$ 
is finite.) On this space, the inner product is given by the right side of (88),

$$
<\phi \mid \phi^{\prime}>=\int \prod_{k, \pm} d F^{ \pm}(k) e^{\hbar G \int d^{3} k|k|\left(\left|F^{+}(k)\right|^{2}-\left|F^{-}(k)\right|^{2}\right)} \overline{\phi\left(F^{ \pm}\right)} \phi^{\prime}\left(F^{ \pm}\right)
$$

The vacuum state by the right side of (99),

$$
\phi_{o}\left(F^{ \pm}\right)=e^{-\hbar G \int d^{3} k|k|\left|F^{+}(k)\right|^{2}},
$$

the operators $\hat{h}^{ \pm}(k)$ act via the right side of (81) and

$$
\hat{h}^{ \pm}(k) \cdot \phi\left(F^{ \pm}\right)=2 \hbar G F^{ \pm}(k) \phi\left(F^{ \pm}\right)
$$

while the operators $\hat{B}^{ \pm}(k)$ act via the right side of 82 )

$$
\hat{B}^{ \pm}(k) \cdot \phi\left(F^{ \pm}\right)= \pm \frac{|k|}{G} \frac{\delta}{\delta F^{ \pm}(-k)} \cdot \phi\left(F^{ \pm}\right) .
$$

The problem is that of transferring this information to the loop space. The strategy we adopted in section 3.2 was to use the form factors $F^{a}\left(k, \gamma^{i}\right)$ to map the loop space $\mathcal{H} \mathcal{L}^{3}$ into the space $\mathcal{F}^{3}$ spanned by triplets of vector fields $F^{a i}(k)$ in the momentum space, and "pull back" the functionals $\phi\left(F^{a i}(k)\right)$ on $\mathcal{F}^{3}$ to functionals $\psi(\vec{\gamma})$ on the loop space via $\psi(\vec{\gamma})=\phi\left(F^{a i}(k)=F^{a}\left(k, \gamma^{i}\right)\right)$. As remarked earlier, however, under this map the image of $\mathcal{H} \mathcal{L}^{3}$ in $\mathcal{F}^{3}$ has no intersection with the space $\mathcal{F}_{o}^{3}$ since the integrals $\int d^{3} k|k|\left|F^{ \pm}(k, \vec{\gamma})\right|^{2}$ diverge for all non-trivial loops $\vec{\gamma}$. Therefore, the pull-back of the n-graviton states $\phi\left(F^{ \pm}(k)\right)$ to the loop space vanishes identically. The idea now is to modify the mapping from the loop space $\mathcal{H} \mathcal{L}^{3}$ into $\mathcal{F}^{3}$ by thickening the loops in an appropriate fashion.

Let us fix an universal averaging function $f_{r}(y)$, such that its integral over the three-dimensional space is 1 and that it goes to a delta function when $r \rightarrow 0$. For concreteness, we make the following choice:

$$
f_{r}(y)=\frac{1}{(2 \pi)^{3 / 2}} e^{-\frac{y^{2}}{2 r^{2}}}
$$

Given any loop $\gamma$ we consider the loop $\gamma+y$ obtained by rigidly shifting the loop by a distance $y$ :

$$
(\gamma+y)^{a}(s)=\gamma^{a}(s)+y^{a}
$$


and we average over $y$ using the weight $f_{r}(y)$. In particular, we define the "average" of the distribution with support on the loop defined in equation (46) as

$$
\begin{aligned}
F_{r}^{a}[x, \gamma] & =\int d^{3} y f_{r}(y) F^{a}[x, \gamma+y] \\
& =\int d^{3} y f_{r}(y) \oint d s \dot{\gamma}^{a}(s) \delta^{3}(x-\gamma(s)+y) \\
& =\oint d s \dot{\gamma}^{a}(s) f_{r}(x-\gamma(s)) .
\end{aligned}
$$

Its Fourier transform $F_{r}^{a}[k, \gamma]$ is by definition the averaged form factor. It is easy to verify that this turns out to be the product of the standard form factor and the Fourier transform of $f_{r}$ :

$$
F_{r}^{a}[k, \gamma]=e^{-\frac{r^{2} k^{2}}{2}} F^{a}[k, \gamma]
$$

the averaging on the small tube in coordinate space corresponds to a damping factor for high momenta. We note that since for any (finite, smooth) loop $\gamma$, the averaged form factor $F_{r}^{a}[k, \gamma]$ is the Fourier transform of a smooth vector field with compact support, the integral $\int d^{3} k|k|\left|F_{r}^{a}[k, \gamma]\right|^{2}$ converges. This technical difference between the averaged form factors and the "bare" form factors introduced earlier plays the key role in what follows.

We now use these averaged form factors $F_{r}^{a}[k, \gamma]$ to define a map from $\mathcal{H} \mathcal{L}^{3}$ into $\mathcal{F}^{3}$ in the obvious fashion: $[\vec{\gamma}] \mapsto F^{a i}(k):=F_{r}^{a}\left[k, \gamma^{i}\right]$. Denote by $\mathcal{H}_{F}$ the (pre-)Hilbert space of all functionals $\phi\left(F^{ \pm}(k)\right)$ on $\mathcal{F}_{o}$ which have finite norm under (88). These functionals can now be pulled back to $\mathcal{H} \mathcal{L}^{3}$. Denote by $\mathcal{H}_{L}$ the space of functionals $\psi_{r}(\vec{\gamma})$ so obtained on the loop space; $\psi_{r}(\vec{\gamma})=\phi\left(F_{r}^{ \pm}(k)\right)$ for some $\phi \in \mathcal{H}_{F}$. This is the required space of physical loop states. Thus, now, in the loop representation, the vacuum is given by:

$$
\left(\psi_{r}\right)_{o}(\vec{\gamma})=e^{-\hbar G \int d^{3} k|k|\left|F_{r}^{+}[k, \vec{\gamma}]\right|^{2}}
$$

Now, the integral in the exponent is manifestly finite and the vacuum wave functional is well-defined on the entire loop space. In the terminology used in section 3.6, the averaging procedure has replaced the divergent expression (99) for the vacuum loop functional by the well defined loop functional

$$
\psi_{r 0}[\vec{\gamma}]=e^{-l_{l_{P}}^{2} \sum_{i, j} \oint d s \oint d t \dot{\gamma}_{i}^{a}(s) \dot{\gamma}_{j}^{b}(t) G_{a b i j}^{(r)}\left(\gamma_{i}(s)-\gamma_{j}(t)\right)},
$$


where

$$
\begin{gathered}
G_{a b i j}^{r}(x)=\int \frac{d^{3} k}{(2 \pi)^{3}} e^{\imath k \cdot x} G_{a b i j}^{r}(k), \\
\tilde{G}_{a b i j}^{r}(k)=e^{-r^{2} k^{2}}|k|\left[\delta_{i j}-\frac{k_{i} k_{j}}{k^{2}}-\imath \epsilon_{i j l} \frac{k^{l}}{|k|}\right]\left[\delta_{a b}-\imath \epsilon_{a b c} \frac{k^{c}}{|k|}\right] .
\end{gathered}
$$

Indeed, this is the only change which is brought about by the averaging procedure. The wave functionals of 1-graviton states are now essentially the same as before; the only difference is that the form factors are replaced by their average values. The inner products between these states are all manifestly finite since all the momentum integrals involved converge. Next, let us consider the physical operators $\hat{h}^{ \pm}(k)$ and $\hat{B}^{ \pm}(k)$. Their expressions are again essentially the same; the only change is that in the action (81) of the graviton operators, the form factor is replaced by its averaged value and in the action (82) of the magnetic field operators, the functional derivative is evaluated at the averaged form factor.

Thus, for each finite, non- zero value of $r$, we have a well-defined representation of the quantum field theory in which states arise as functionals on the loop space. There are no infinities and, irrespective of the choice of the averaging parameter $r$, the representation is entirely equivalent to the standard Fock representation. There is no cut-off involved and the loop description captures gravitons with arbitrarily high frequencies. In any calculation of a physical quantity, the dependence on $r$ cancels out. Thus, $r$ is not a regularization parameter.

\section{Discussion}

We have completed the main goal of this paper, which is the construction of the Fock space of graviton states, together with inner product and physical observables, in terms of the loop quantization. The vacuum is given by the loop functional (118), the n-graviton states are given by the loop functionals obtained by multiplying the vacuum with powers of $F_{r}^{+}[k, \vec{\alpha}]$ and Hermite polynomials in $F_{r}^{-}[k, \vec{\alpha}]$. The inner product is given by the expression (117). For the physical states this expression reduces to Gaussian integrals of polynomials. The Hilbert space of states $\mathcal{H}$ is formed by the loop functionals $\psi_{r}[\vec{\alpha}]$ which are normalizable linear combinations of the n-graviton states. 
The structure that we have obtained is isomorphic to the standard Fock space of gravitons.

We conclude with a few remarks.

- In the construction of the theory we have used a somewhat novel quantization procedure: the connections $A_{a j}$ are self-dual rather than of positive frequency and the inner product on the physical states is selected using the reality condition. The inner product so determined turns out to be the standard Poincaré invariant one in spite of the fact that Poincaré invariance is never explicitly invoked. These features are important from the viewpoint of full quantum general relativity where we cannot define positive frequency fields nor do we have an access to the Poincaré group.

- Two peculiar features of the loop representation of linearized gravity are the surprising chiral asymmetry of the formalism and the use of triplets of multiple loops. The first is a consequence of the chiral asymmetry of the new canonical framework; the second is the key technical idea that allowed us to construct the theory.

-Note that the Planck length $l_{P}$ remains a parameter in the quantum theory of free gravitational waves. This is not surprising. The situation is analogous to that in particle mechanics where, although the mass does not appear in the classical equations of motion of a free particle, the Compton wave-length is an important parameter in the corresponding quantum theory.

-The averaged representation depends on an arbitrary parameter $r$. (More precisely, it depends upon an arbitrary function $f_{r}(x)$.) Physical quantities do not depend on this parameter. The physical meaning, if any, of this parameter remains unclear. Had we worked with positive frequency connections rather than self dual, for example, the averaging would not have been necessary. Recall also that, unlike in the Maxwell theory, wave function renormalization does not remove divergences in the present description of linearized gravity. This difference is, in turn, due to the fact that the linearized theory of gravitons still contains a dimensional parameter, the $l_{P}$ length. As a result, the momentum integrals in the ground state wavefunctionals (and inner product) are now two powers of momentum more divergent than they are in the Maxwell theory. These facts are, of course, related to the nonrenormalizability of the standard perturbation theory for general relativity. In the loop representation geared to self-dual connections, they seem to have consequences already at the linear level. 
- The loop representation is equivalent to the Fock representation for linearized gravity. Hence, if we attempt to construct the conventional perturbation theory using the framework of this paper, we would find the same results as in any other treatment of the Fock states. The potential interest of our results is in another direction. From this standpoint, the relevant question is the following: Is the Fock space of gravitons the correct linearization of the full quantum theory of general relativity? In the full non-perturbative quantization of general relativity there should be states that represent classical field configurations, at least at large scales. This follows from the correspondence principle. However, there is no principle that guarantees that these exact quantum states that look semi-classical at large scales continue to behave semiclassically at the Plank scale. If the full quantum theory implies that at short distances there is no semiclassical behavior, then we should expect that the quantization of the linear theory around a flat background space does not make sense at short distances. Since in the loop representation we have solutions of the full non-perturbative quantum constraints [3] and since we now understand the linearized theory in the loop representation, it should be possible to investigate this issue in detail. Work is now in progress along these lines [19].

A.A. and L.S. thank the Department of Physics of the University of Trento, and in particular Marco Toller, for the hospitality during the period in which this work was completed. This research was supported in part

by the NSF grants INT88 15209, PHY90 16733 and PHY90 12099 and by research funds provided by Syracuse University.

\section{References}

[1] A. Ashtekar, Phys. Rev. Lett. 57, 2244 (1986); Phys. Rev. D36, 1587 (1987).

[2] T. Jacobson and L. Smolin, Nucl. Phys. B299, 295 (1988).

[3] C. Rovelli, L. Smolin, Nucl. Phys. B133 (1990) 80; Phys. Rev. Lett. 61, 1155 (1988). 
[4] A. Ashtekar, Lectures on Non-perturbative canonical gravity, notes prepared in collaboration with R.S. Tate (World Scientific, Singapore, 1991).

[5] C. Rovelli, Ashtekar formulation of general relativity and looprepresentation of quantum gravity: a report, Class. and Quant. Grav. (in press).

[6] R. Gambini and A. Trias, Phys. Rev. D23 553 (1981); Lett. al Nuovo Cimento 38, 497 (1983); Phys. Rev. Lett. 53, 2359 (1984); Nucl. Phys. B278, 436 (1986); Phys. Rev. D39, 3127 (1989); R. Gambini, Loop space representation of quantum general relativity and the group of loops, Preprint University of Montevideo, (1990).

[7] A. Ashtekar, V. Husain, C. Rovelli, J. Samuel and L. Smolin , Class. and Quan. Grav. L185-L193 (1989).

[8] A. Ashtekar and R.S. Tate, An extension of the Dirac program for quantization of constrained systems: Examples, in preparation (1991).

[9] A. Ashtekar and C. Rovelli, A loop representation for the quantum Maxwell field, Syracuse preprint (1991).

[10] C. Rovelli and L. Smolin, Loop representation for lattice gauge theory, Pittsburgh and Syracuse preprint (1990).

[11] A. Ashtekar, J. Math. Phys. 27, 824 (1986); A. Ashtekar, C. Rovelli and L. Smolin, Self duality and quantization, J. Geo. \& Phys. (in press).

[12] R. Penrose and W. Rindler, Spinors and spacetime, Vol. 2 (Cambridge University Press, Cambridge 1986).

[13] C. Kozameh, W. Lamberti and T. Newman, Holonomies and Einstein Equations, (to appear in Ann. of Phys. 1991).

[14] A Ashtekar and J. Lee, Weak field limit of general relativity: A new Hamiltonian description. Syracuse preprint (1991).

[15] A. Ashtekar, L. Smolin and C. Rovelli, Self duality and quantization of linearized gravity, in preparation (1991). 
[16] L. Smolin, The $G_{\text {Newton }} \rightarrow 0$ limit of Euclidean quantum gravity, in preparation.

[17] A. Ashtekar (with invited contributions), New Perspectives in Canonical Gravity, (Bibliopolis, Napoli, Italy, 1988).

[18] C. Rovelli, Time in quantum gravity: Physics beyond the Schrödinger regime, Universita' di Roma preprint (1988); Phys. Rev. D43, 442 (1991).

[19] A. Ashtekar, C. Rovelli and L. Smolin, in preparation (1991). 